Article

\title{
The Urgent Need of a Naturalized Logic
}

\section{Lorenzo Magnani (iD}

Philosophy Section and Computational Philosophy Laboratory, Department of Humanities, University of Pavia, Corso Str. Nuova, 65, 27100 Pavia PV, Italy; Imagnani@unipv.it

Received: 14 October 2018; Accepted: 20 November 2018; Published: 11 December 2018

\begin{abstract}
The naturalization of logic aims at a revision of mainstream logic. In this article, I contend it is an urgent task to be completed. This new project will permit a new collaboration between logic and cognitive science. This can be accomplished doing for logic what many decades ago Quine and other philosophers undertook in the case of epistemology. First of all, this article analyzes how the naturalization can be achieved thanks to some insights provided by the recent John Woods' book Errors of Reasoning: Naturalizing the Logic of Inference; important concepts that regard a naturalized logic are synthetically analyzed: errors (and the problem of fallacies), paradigm creep, third-way reasoning, consequence-having and consequence drawing, agent based reasoning. The article also takes advantage of my own studies, which are aimed both at exculpating the negative fallacious character of abduction (it is the fallacy of the affirming the consequent) and at illustrating the EC-model (Eco-Cognitive model) of it, I have recently proposed. Aiming at encouraging the project of naturalization of logic, the article specifically recommends the increase of logical research on abduction, and emphasizes how current philosophical and logical research on human inferences is indebted towards Charles Sanders Peirce, a philosopher whose importance and modernity are too often underestimated. The final part of the article will introduce an analysis of the importance of the so-called optimization of situatedness, a concept that is necessary to understand that maximization of "abducibility", which characterizes modern science.
\end{abstract}

Keywords: abduction; agent-based reasoning; creativity; eco-cognitive model; eco-cognitive openness; fallacies; errors of reasoning; third-way reasoning; naturalization of logic

\section{Naturalization, Cognitive Errors, and Logic}

Dealing with the importance of cognitive errors commonly committed by human beings immediately refers to the need of a new process of naturalization of logic. ${ }^{1}$ If we aim at delineating this project of naturalization of logic first of all we have to state the extreme importance of cognitive science and of its commitment to empirical research: of course I am here referring to those empirical observations which regard how people reason in various contexts (also empirical studies of argumentation in context are of course important). Moreover, to the aim of clarifying the main aspects of this process the case of abductive reasoning is exemplar, as I will address in the second part of this article. A research agenda would have to be created for empirical-based research on the topic.

The idea of naturalization has been already important in philosophy: Dewey [2], Toulmin [3], Quine [4], and Finocchiaro [5] (pp. 6-7) already stressed its importance and capacity to innovate ways of thinking against the excess of speculations and idealizations. Just to refer to the two important authors of the modern times, the naturalization of logic is the grounding idea of Dewey's experimental

1 The book Errors of Reasoning: Naturalizing the Logic of Inference, by John Woods [1], depicts a well-defined program of naturalization of logic, also thanks to a strong attention to the importance of the role of cognitive science in this process. 
logic: "Logic is a social discipline [...] [E]very inquiry grows out of background of culture and takes effect in greater or less modification of the conditions out of which it arises. Merely physical contacts with physical surroundings occur. However, in every interaction that involves intelligent direction, the physical environment is part of a more inclusive social or cultural environment" [2] (vol. 12, p. 27). Toulmin too contends that logical has to become "more empirical": "A similar theme is present in the early writings of Toulmin: Logic [...] may have to become less an a priori subject than it has recently been [...]. Not only will logic have to become more empirical; it will inevitably tend to be more historical [3]" (p. 257).

Moreover, the idea of a naturalization of logic has been already promoted thanks to the fecund interplay between formal tools and the new twenty century studies in the area of artificial intelligence (AI) and cognitive science. For example, Kowalski's book Computational Logic and Human Thinking [6] lists and illustrates various types of reasoning (for example deduction, induction, abduction, nonmonotonicity, planning, decision making, temporal and meta-reasoning), and de facto realizes an extension of logic in action favored by research in computer science and AI. In addition, Stenning's book Human Reasoning and Cognitive Science [7] furnishes new ideas regarding the naturalization of logic which stresses the need for finding new logical systems able to account for how actual people reason. ${ }^{2}$

In the book Errors of Reasoning, Woods further emphasizes the importance of the so-called "empirical sensitivity": a naturalized logic has to take into account the results provided by cognitive science and at the same time it has to carefully check every difference that there is between the mainstream logical inferential standards and the findings of cognitive and other sciences. In summary, "It is not in the general case preferable-indeed it is not smart and not even possible—to upgrade our cognitive targets in ways that favor truth-preservation or experimental/statistical confirmation as general cognitive strategies" [1] (p. 198). In this perspective, naturalizing logic is the refusal of all kinds of normative "presumption", and it is indeed in this sense, as I have stressed above, that the naturalization of logic is in tune with the studies which characterize the AI tradition.

Abandoning the usual tendency to build formal systems, idealized, the novel naturalized logic favors the need of carefully considering the actual aspects of human reasoning, postponing the "obsession" regarding judgments of goodness or badness or at least submitting those judgments to the default rule called "Convergence of the Normative on the Normal" (NN-convergence principle): the rule states that, in the absence of special reasons to the contrary, humans reason well when they reason in the manners that humans standardly do reason in for the circumstances of actual life. The $N N$-convergence principle is not a safe default for all aspects of human reasoning; its use here is only referred to premiss-conclusion reasoning. It suggests to us that a human agent's premiss-conclusion inference is the correct manner to reason when his conclusion-generating cognitive instrumentation is in a good state and, and it happens in the right way thanks to an operation on good information and without the contrary effect of uncongenial adversities.

We perfectly know that the mainstream logical tradition is oriented by the so-called mathematical turn in logic that occurred in the last decades of the 19th century, with the birth of mathematical logic. The emphasis on the role of cognitive science offers a new perspective, which departs from the ideality furthered by classical logic to something open to the consideration of agents, goals, resources, actions and to the role of time. Of course, these new tasks will generate the need for an improvement of the already available formal technicalities, to build new ones more appropriate to the naturalization, thus possibly avoiding excess of complication. Updates will instead have to look for simplification,

2 In my article [8], I have also illustrated in detail other research that can be seen as connected to the naturalization of logic: it mainly consists of AI studies that take advantage of logical modeling-promoted by Luís Moniz Pereira-in counterfactual reasoning [9,10], moral reasoning [11-13], mutual debugging and argumenting [14,15]; objecting [16,17], preferring, forgetting, updating, intention recognition and decision making. Also the studies regarding evolutionary game theory in the case of emergent population norms and emergent cooperative moral behavior are related to the need of nuturalizing logic, considering agents belonging to populations and groups, and certainly point out fundamental issues which help to overcome the expressive inflexibility of the conventional logical systems [18-20]. 
and we can also provocatively advance the following idea: the empirical turn in logic can also constitute a new effort to change some methodological habits, as Woods suggests, moderating the idea that it is a duty of the theorist to demonstrate theorems; after all, theorem-proving is not required for population biology; consequently, why, asks Woods, should it be requested in the case of a naturalized logic?

\section{2. "Paradigm Creep" and "Third-Way" Reasoning}

The new naturalistic approach is especially suitable to shed a new light on some central issues that refer to the current epistemologico/cognitive debate. Woods [1] (p. 481) contends that we are dealing with a kind "paradigm creep", related to the puzzling problem of the importation of mathematical instrumentation into scientific research about cognition. Woods' perspective could be summarized in the proposition "Do not for your present purpose employ successful methods", in other words: it is not said that methods that optimally work in a determinate field can automatically be good elsewhere. In this perspective, it is clear that, in human reasoning, particularly when consisting in a movement from premisses to conclusions, it is unlikely and uncommon that the usual criteria of deductive validity or statistico-experimental inductive power are ever at work. New criteria for what Woods calls "third-way" reasoning [1] (Chapter 7) have to be built taking advantage of the already available models regarding nonmonotonic, default, ceteris paribus, agenda-relevant, inconsistency-adaptive, abductive reasoning, for which neither common deductive validity nor inductive force are appropriate as good criteria of assessment. A great part of human "right" reasoning is third-way reasoning, and this rightness is reached thanks to the exploitation of requisites that are different from the standards of deductive validity (or inductive force).

I have just said in the previous paragraph that it is relatively unlikely that the standards of statistico-experimental inductive strength can be at work: Bayesianism is very well-known in current cognitive science: what is the consequence of the new perspectives on naturalizing logic with respect to Bayesian models of cognition? I agree with the skeptical attitude adopted by John Woods: Bayesianism, as an appropriate way of representing normatively assessable premiss-conclusion inferences as executed by human beings in the situations of everyday actual life, has to be rejected. A summary of various argumentations against Bayesianism — and in general against all normatively idealized models to human cognitive performances-is still illustrated in Woods' book [1] (Chapter 2 and pp. 75, 80, 142) and can be simplified as follows. As used to represent actual human reasoning performances-in the cognitive economies in which humans live out their lives-Bayesianism's abstract and ideal normative rules cannot be implemented mainly because their computational intractability for human beings. Norms, such as the closure of belief under consequence, are not only false of human actual praxis but transfinitely false of it. Humans, even when they are performing cognition in their own possible best way, cannot arrive close to this ideal norms in any finite degree. This huge gap is usually explained away by saying that we humans are very "bad" at reasoning.

Usually, Woods says, two argumentations are proposed to the aim of explaining the prescriptive authority of Bayesian rules, as described in [1] (Chapter 2). First, it is said that the potency of those rules derives from the truths (or analyticity) in-the model-second, that it derives from the "necessity" that is generated by their mathematical expression (given the fact that we in general accept the idea that mathematical truths are necessary). It is patent that they are very poor arguments. There is a third argument, more complex but certainly inadequate too. The argument resorts to, say, taking advantage of a kind of reflective equilibrium demonstration, that the prescriptive authority of the Bayesian norms are prescriptively binding on human individual behavior when they are clearly mirrored by the standard praxis of the communities in a broad way. The problem is: how can these communities be determined? Are they the everyday ones or do they coincide with the people who conduct studies on human reasoning, i.e., are they Bayesian themselves? In the case of this last argumentation, we still obtain a bad result because these expert people, from the point of view of their ways of reasoning, are not different from the rest of human beings and, moreover, in terms of what they consider good 
reasoning performances, a degree of conformity to their own prescriptive rules can emerge (but this is an obvious consequence!).

I have anticipated that abduction is a wonderful example of "third-way reasoning" and it is in need of a naturalization of logic: Bayesianism could be the solution because it is also at play when dealing with abductive cognition, and it is often preferred by some logicians and by some cognitive scientists who work on abduction. Still, the problem is that Bayesianism first of all does not present a proved (or even plausibly guessed) prescriptive legitimacy. Second, we have to recall that Peirce correctly maintained that a rationally optimal human abductive cognition is not related to the objective probability of the hypothesis at stake and, at the same time, should not raise its subjective probability. Consequently, enforcing Bayesian rules with the intention of saying the last word about abduction involves a betrayal of Peirce's ideas: of course, Bayesianism provides technical tools able to help us to perform abductions just offering an ideal method, among many others, that can occasionally be used (for example in AI). Indeed, the reader does not have to misunderstand me: it is certainly desirable that Bayesianism should be made more realistic by dropping the idealized condition of closure of belief under logical consequence, but this does not mean that having some standards of good inductions and abductions does not have to still be desirable too.

\section{3. "Consequence-Having" and "Consequence-Drawing"}

When dealing with a project of naturalization of logic, we have to distinguish between "consequence-having", which is related to the "logical space" concerning the usual concept of "logical consequence" of the mainstream logical tradition, and "consequence-drawing", which refers to the "the reasoner's mind" in a non-idealized space, pragmatically influenced. Consequently, consequence-drawing consists of a pragmaticization of consequence-having. Nonmonotonic and abductive logics already available have provided a naturalized account of various kinds of third-way reasoning: however, a stronger further naturalization will be intertwined with a reshaping of them to the aim of grasping more aspects of the conclusion-drawing structural relationships in various particular contexts. Indeed, Woods stresses that the conclusion-drawing is basically a special kind of inference that is referred to a cognitive agent $X$, the information $I$ the agent reasons on, a background database $\Delta$ of information that is at disposal of the agent, a cognitive agenda $A$ of duties for the agent, a conclusion $\alpha$, derived after the application of the background $\Delta$ to $I$, and a disposition $D$ to answer-if asked-justification requests for $\alpha^{\prime}$ s being drawn by citing $I(\Delta)$ [1] (pp. 285-286).

Naturalizing logic is certainly the improvement of the already available agent-based logics, probabilistic systems, belief-change and decision theories, epistemic and justification logics, and fallacy theory, but also the revision of some aspects of discourse analysis and argumentation and of normative psychology. However, a deep revision of the fallacy theory is still needed for the aim of exculpating "errors of reasoning" by showing their virtues and positive characters. To this aim, in the quoted book, Woods further promotes new perspectives on defeasible and default reasoning, nonmonotonic systems, autoepistemic and anti-closed world belief-formation, the role of presumption in the government of belief, etc. An interesting chapter also regards in a naturalized way the communication of knowledge when one individual tells something to another. In addition, abduction-see below in Section 6-a basic inference of human and animal life, is one of the main issues of the book.

In summary, as a new ambitious project, naturalizing logic aims at contrasting the priority of the prescriptive characters typical of the mainstream logic of premiss-conclusion reasoning, also taking advantage of the interplay between logic and the the new sciences of cognition. Here, the role of the relationship between cognitive science and empirical research has to be further emphasized, stressing that we are not referring here to empirical research merely interested in abstract models of the mainstream tradition, but to a much wider area of human cognitive behaviors. In this perspective, abductive reasoning is a good example, given the fact that this kind of reasoning cannot be satisfactorily studied in the framework of classical logic and, at the same time, refers to a broad area of human cognitive performances, which can only be ascertained empirically. 


\section{Agent-Based Pragmatically Oriented Logics}

Peirce considered it unlikely to be able to build an agent-based "logic" and consequently-for example-a logic of human abductive cognition, pragmatically oriented. Indeed, Peirce thinks that logic (and scientific reasoning) and practical reasoning have to be clearly distinguished (it is impossible to imagine a practical logic in general and a fortiori a logic of abductive cognition): "In everyday business reasoning is tolerably successful but I am inclined to think that it is done as well without the aid of theory as with it" [21] (p. 109). "My proposition is that logic, in the strict sense of the term, has nothing to do with how you think [...]. Logic in the narrower sense is that science which concerns itself primarily with distinguishing reasonings into good and bad reasonings, and with distinguishing probable reasonings into strong and weak reasonings. Secondarily, logic concerns itself with all that it must study in order to draw those distinctions about reasoning, and with nothing else" (ibid., p. 143). It is curious that the founder of the first studies on abductive reasoning is not at the same time inclined to consider the possibility of what in this article I call "naturalization of logic".

Naturalizing logic overcomes Peirce's skepticim: a new naturalized logic is legitimate and certainly has to be agent-based. The particular example of a new naturalized logic of abduction is important because it also refers to the importance of showing the virtues of the well-known fallacy "affirming the consequent" - which indeed classically models abductive reasoning. Peirce's abduction is illustrated as both (a) a surrender to an idea, and (b) a way for examining/testing its consequences: these aspects certainly mirror in themselves some basic aspects of practical reasoning. Woods contends that already available logics of abduction [1] (Chapter 11) are still excessively idealized illustrations of the corresponding cognitive conduct of a human actor. To overcome this weakness, I myself have delineated the so-called eco-cognitive ${ }^{3}$ model of abductive reasoning in [22]: the abductive human performer is naturalistically framed in the perspective of the distributed cognition tradition.

Given the fact, as Gabbay and Woods maintain, that a logic is a symbolized idealization of a kind of actor as a logical agent, which characters of a human agent will a logic of abduction take into account? Following these authors, it seems that an actual human agent is a kind of organic actualization of a nonmonotonic paraconsistent base logic: it is certain that the strategies furnished by classical logic and some closely related non-standard logics compose a tiny part of the cognitive capacities of a single person. The main reason is that real human agents usually are not committed to avoid errors like "classical" idealized logical agents. I repeat, Gabbay and Woods contend that a formal model is an idealized illustration of the performances of an actual agent and so it presents itself as very distant with respect to the empirical data that reflect a human reasoner: for these patent reasons, it is obvious to state that in the logic that represents a practical agent (for example abductive) problems of "contextual" - for example-relevance and plausibility are fundamental.

Moreover, we have to remember that a "real" human abductive agent runs at both conscious and unconscious levels, and at both levels he is committed to (or is oriented by) consider the truth conditions on propositional frameworks, to state conditions on belief structures and their stabilization, and also to groups of rules embedded in various argumentative frameworks, for example for assessing arguments. These three aspects cut through explicit and implicit cognition. I have to add that the majority of the central reasoning capabilities of a human actor are enriched by a "story", and are in turn related to the several propositional relations he detects in his cognitive environment and which he considers relevant, with several cognitive reasons to modify his mind or to reason in an alternative way, and with various motives to distribute different strategies of argumentation.

Naturalized logical systems have to be taken as mimetic: in this sense, they are "mimetic representations" (for example, nonmonotonic systems are certainly more able than classical logic to "mime" —an so they are more psychologically veridical—human beings' actual cognitive performances). Classical logical agents certainly embody ideals of "good reasoning"; unfortunately, in the perspective of

3 I will illustrate the significance of this expression below, in Section 6.2. 
a naturalized logical perspective, good reasoning is always good in relation to a target or an agenda which may also be tacit. In this sense, reasoning validly is never in itself something that leads to good reasoning; Gabbay and Woods say:

It is that the reasoning actually performed by individual agents is sufficiently reliable not to kill them. It is reasoning that precludes neither security not prosperity. This is a fact of fundamental importance. It helps establish the fallibilist position that it is not unreasonable to pursue modes of reasoning that are known to be imperfect "Given the cognitive goals typically set by practical agents, validity and inductive strength are typically not appropriate (or possible) standards for their attainment" [23] (pp. 19-20, 25).

I have anticipated that abduction in the framework of classical logic is the fallacy of affirming the consequent, and so it is a kind of reasoning that can be affected by mistakes and failures, sometimes seen as an example of imperfect reasoning: at the same time, we all know that it is a kind of a very useful reasoning because we cannot imagine the prosperity of our civilizations without linking it to the extraordinary capacity of humans to guess good hypotheses of various kinds. Human agents are hasty inducers/generalizers, bad predictors, and hybrid abducers unlike ideal (logical and computational) agents: the naturalization of logic is devoted to illustrate and describe the force and the fruitful side of these inferential routines, otherwise considered "impaired".

\section{5. “Redeeming" Fallacies}

I contend that from Aristotle onwards logic has always treated fallacies in an excessive negative way. It would be better for logic to rethink fallacious reasoning in a novel perspective and the naturalization of logic is surely the correct method able to pursue this target. In this perspective, Woods's book Errors of Reasoning represents a kind of "manifesto" of the naturalization of logic: it exactly states the two main preconditions of the project, the need of both (1) redeeming fallacies and (2) criticizing the excess of prescriptive authority contended by formal models of ideal reasoners. In this framework, we can see fallacies not only as active, possibly in a negative way, both in everyday and scientific reasoning, but, also, the facto, as the occasional best tool for arriving to reliable cognitive results. These results are able to grant to human beings adequate fitness in everyday life but also consequent adaptive effects in more complicated inferential activities, such as in the case of scientific discovery and modeling.

The positive cognitive role of classical deductive and inductive fallacies such as ad baculum, ad hominem, ad populum, ad ignorantiam, ad verecundiam, affirming the consequent, denying the antecedent, hasty generalization, equivocation or quaternio terminorum, the gambler's fallacy and post hoc ergo propter hoc, has to be vindicated. Reasonings of this type have a pervasive presence in cognitive contexts and, as already said, often facilitate the reaching of crucial general rational cognitive ends. Similarly, Peirce said that humans' abductions are-and the reason is fundamentally evolutionary_-"akin to the truth" [24] (7.220): ${ }^{4}$ they work positively in various situations and actually consent good survival and successfulness. We have to abandon the old view that exclusively sees a fallacy as a mistake in reasoning, a mistake which is extremely frequent in human arguments and usually also effectively deceptive. Just to make an example, fallacies such as hasty generalization and ad verecundiam are classified as "inductively" weak inferences, and affirming the consequent is a deductively invalid inference. The problem is that, when they are exploited by real reasoners, that is in an eco-logical ${ }^{5}$ and not only logical—that is "ideal" and abstract—way, they are no longer fallacies, but instead productive ways of inferencing important cognitive conclusions. ${ }^{6}$

\footnotetext{
See also below, p. 9.

That is when fallacies are considered in an actual human and social flux of information and/or speech-acts.

Further illustration of the so-called "EAUI-conception" of fallacies (fallacies are Errors, Attractive, Universal, and Incorrigible) is provided in the already quoted Woods" book [1] (p. 136).
} 
In [25], I have delineated a clear differentiation between strategic and cognitive rationality: in this perspective, various fallacies can be considered at the same time cognitive mistakes and strategic successes, that is, it happens that it is more appropriate (and sometimes rational) to go ahead strategically, even if we are committing a "logical" mistake (it is, for example, the case of hasty generalization, often useful in circumstances of real life): ${ }^{7}$ in these cases, we can affirm we are dealing with the so-called "casual" truth preserving aspect of fallacies. ${ }^{8}$

It is important to note that, according to Woods (and I agree with him), it is not that it is often "strategically" excused to adopt fallacies, but rather that traditional fallacies simply are not fallacies. This consideration is insidious: we can clarify it by saying that the traditional conception of fallacies is embedded in a deep aristocratic idea of human thinking that neglects its deep eco-cognitive aspects. These cognitive acts that are based on fallacious inferences patently show that cognition can be good and fruitful even in front of limited information and knowledge and when sound inferences lack. In this light, a more profound and reliable knowledge and sound inferences are no more at the center of the attention, instead reserved to them by the philosophical, epistemological, and logical received views. After all, reliable belief is sufficient for human collectives to realize a good fitness, as they do, and, in this sense, belief seems more "economical" than completely attained knowledge.

\section{Naturalizing the Fallacy of Affirming the Consequent: Abduction in an Eco-Cognitive Perspective (the EC-Model)}

A lot of studies on abduction produced in several areas of research, such as logic, cognitive science, philosophy, and AI, have already vindicated the cognitive relevance of the the so-called fallacy "affirming the consequent" (I have already said that in the framework of the classical logic abductive reasoning is rendered by this fallacy), that is the error of possessing a conditional and its consequent, and from this consequent deriving the antecedent. In reality, this kind of reasoning is very useful, and, when reconsidered in the light of the naturalization of logic, the fallacy is shown to possess an extraordinary cognitive importance, in most of the every-day reasoning contexts, included diagnosis and creative processes, but also in sophisticated situations, for example regarding scientific reasoning. Abduction usually provides good hypotheses, so furnishing an example of what some logicians have called "material validity": 9 the invalid form (the fallacy) provides a cognitive good semantic result. In the last three subsections of this article, I plan to illustrate a naturalization of the fallacy of the affirming the consequent: it is in this way that we can detect and stress the cognitive very positive aspects of abduction, and, to this aim, I will adopt and further delineate the so-called Eco-Cognitive Model (EC-model) of abduction that I have recently proposed.

\subsection{Ignorance-Preservation and Abduction}

In my last book on abductive cognition [32] (Chapter 1), taking advantage of Gabbay and Woods' ideas, I have described that abduction is an inferential process in which something that lacks epistemic virtue is used and assumed because it has virtue of another kind. For example: "Let $S$ be the standard that you are not able to meet (e.g., that of mathematical proof). It is possible that there is a lesser epistemic standard $S^{\prime}$ (e.g., having reason to believe) that you do meet" [1] (p. 370). Abduction, in the light of the naturalized logic delineated in the previous sections, and extracted from the fallacious destiny that the mainstream logic assigned to it, has to be fundamentally seen as a scant-resource strategy [23], which goes forward in absence of knowledge and exhibits an ignorance-preserving

7 Various concrete examples are illustrated in [26].

8 Even if they are not committed to build naturalized logical models of reasoning Gigerenzer et al. [27-30] emphasized the "fast and frugal heuristics", and their strategic role in reasoning ("strategic rationality" they say) as tools that can solve various problems in settings characterized by limited knowledge and time. In these studies, strategic reasoning is seen, in a kind of evolutionary perspective, as an adaptive toolbox, related to an ecological and social view.

9 The concept of material validity is explained by Brandon [31], as a situation in which we face with a semantically valid inference, even if it instantiates an invalid syntactic scheme. 
(or, better, an ignorance mitigating) character. Of course "[...], it is not at all necessary, or frequent, that the abducer be wholly in the dark, that his ignorance be total. It needs not be the case, and typically isn't, that the abducer's choice of a hypothesis is a blind guess, or that nothing positive can be said of it beyond the role it plays in the subjunctive attainment of the abducer's original target (although sometimes this is precisely so)" (p. 370). Abductive reasoning is the answer to an ignorance-problem: no knowledge is available to solve a problem, so we have three chances (1) looking for new knowledge, appropriate to solve the problem; (2) yielding our ignorance in a kind of provisional capitulation; (3) guessing a hypothesis able to solve the problem and to provide a new basis for action, that is we abduce (in a framework of constitutive ignorance).

In this perspective, Woods proposes a general scheme of an abductive inference that can be formally rendered as follows. Let $\alpha$ be a proposition that characterizes an ignorance problem. $T$ will be the agent's epistemic target with respect to the proposition $\alpha$ at a given time, $K$ his knowledge-base at that time, $K^{*}$ an immediate accessible successor-base of $K$ that regards the agent's means to produce in a timely way, ${ }^{10} R$ the attainment relation for $T, \rightsquigarrow$ the subjunctive conditional relation, $H$ as the agent's hypothesis, $K(H)$ the revision of $K$ thanks to the addition of $H, C(H)$ refers to the conjecture of $H$ and $H^{c}$ its activation. The general schema of and abductive inference abduction can be illustrated as follows (GW-schema): ${ }^{11}$

$\begin{array}{ll}\text { 1. } T ! \alpha & \text { [establishment of } T \text { as an epistemic target with respect to a } \\ & \text { proposition } \alpha \text { ] } \\ \text { 2. } \neg(R(K, T)) & \text { [fact] } \\ \text { 3. } \neg\left(R\left(K^{*}, T\right)\right) & \text { [fact] } \\ \text { 4. } H \notin K & {[\text { fact] }} \\ \text { 5. } H \notin K^{*} & {[\text { fact] }} \\ \text { 6. } \neg R(H, T) & \text { [fact] } \\ \text { 7. } \neg R(K(H), T) & \text { [fact] } \\ \text { 8. If } H \rightsquigarrow R(K(H), T) & \text { [fact] } \\ \text { 9. } H \text { meets further conditions } S_{1}, \ldots S_{n} & \text { [fact] } \\ \text { 10. Therefore, } C(H) & \text { [sub-conclusion, 1-9] } \\ \text { 11. Therefore, } H^{c} & \text { [conclusion, 1-10]. }\end{array}$

The schema seems very appropriate for abduction. It is a given that $H$ neither in the agent's knowledge-set nor in its immediate successor. Given the fact that $H$ is not in $K$, then the revision of $K$ by $H$ is not a knowledge-successor set to $K$. Even so, $H \rightsquigarrow R(K(H), T)$. Consequently, we have an ignorance-preservation, as required (cf. [1] (Chapter eleven)).

Note: Line 9 says that $H$ does not have more plausible or relevant rival constituting a larger degree of subjunctive attainment. Of course, establishing the $S_{i}$ is the most difficult problem for abductive inference: indeed, there are many potential candidate hypotheses. Woods says that this involves, for example, the consistency and minimality constraints. These constraints reproduce the lines 4 and 5 of the standard AKM schema of abduction, ${ }^{12}$ which is illustrated as follows:

$10 K^{*}$ is an accessible successor of $K$ to the degree that an agent has the know-how to construct it in a timely way; i.e., in ways that are of service in the attainment of targets linked to K. For example, if I want to know how to spell "accommodate", and have forgotten, then my target can't be hit on the basis of $K$, what I now know. However, I might go to my study and consult the dictionary. This is $K^{*}$. It solves a problem originally linked to $K$.

11 That is, Gabbay and Woods Schema.

12 This classical representation of abduction is rendered by what Gabbay and Woods [23] call AKM-schema, which is contrasted to their own (GW-schema), which I am just illustrating in this subsection. A refers to Aliseda [33,34], K to Kowalski [35], Kuipers [36], and Kakas et al. [37], and M to Magnani [38] and Meheus [39]. A full description of the AKM schema is contained in [22] (Chapter 2, Section 2.1.3). 
1. E,

2. $K \not \subset E$,

3. $H \not \rightarrow E$,

4. $K(H)$ is consistent,

5. $K(H)$ is minimal,

6. $K(H) \rightarrow E$,

7. Therefore, $H$

[23] (pp. 48-49),

where obviously the conclusion operator $q \rightarrow$ cannot be classically interpreted. The target has to be an explanation and $K(H)$ bears $R^{\text {pres }}$ (that is, the relation of presumptive attainment) to $T$ only if there is a proposition $V$ and a consequence relation $\rightarrow$ such that $K(H) \rightarrow V$, where $V$ indicates a payoff proposition for $T$. In turn, in this schema, explanations are interpreted in consequentialist terms. If $E$ is an explanans and $E^{\prime}$ an explanandum, the first explains the second only if (some authors further contend if and only if) the first implies the second. Of course, we can add that the AKM schema embeds a D-N (deductive-nomological) interpretation of explanation, as I have also detailed in [38] (p. 39).

Finally, in the GW-schema, $C(H)$ is read "It is justified (or reasonable) to conjecture that $H^{\text {" }}$ and $H^{c}$ is its activation, as the basis for planned "actions".

In summary, the GW-schema stresses that $H$ is merely guessed, hypothesized, so that the truth is not assured: $K(H)$ presumptively attains $T$, the agent just "presumes" that his target is now accomplished. Given the fact that presumptive knowledge accomplishment is not an actual knowledge accomplishment, the agent's abduction has to be thought as preserving the ignorance that already generated his (its, if we are dealing with a machine) original ignorance-problem. Consequently, abduction does not have to be considered the "solution" of an ignorance problem, but rather a simple response to it, in which the agent attains presumptive achievement of a cognitive task rather than actual accomplishment. $\mathrm{C}(H)$ means the conclusion that it follows from the facts of the schema that $H$ is a worthy object of conjecture. We have to add that, to the aim of solving a problem, it is not needed that an agent actually can guess a hypothesis, but it is only needed that he expresses that the hypothesis is worthy of conjecture. Moreover, conceiving $H$ justified to conjecture is not equivalent to conceiving it justified to accept/activate it and finally to send $H$ to experimental examination. In this perspective, $H^{c}$ indicates the decision to submit $H$ to further work in the field of enquiry in which the ignorance-problem originated, that is, the activation of $H$ represents a good cognitive basis for acting. Of course, there are many cases in which abduction stops at line 10, that is, no activation is performed: "When this happens, the reasoning that generates the conjecture does not constitute a positive basis for new action, that is, for acting on that hypothesis" [1] (p. 371). When the hypothesis is instead evaluated, we have to note that this process of evaluation and so, of activation, does not have to be considered abductive, but inductive, as Peirce illustrated. ${ }^{13}$ Hence, in the perspective of the GW-schema, testability is not constitutive of abduction.

A last note concerns an obvious characteristic of human abduction that has to be stressed. In the case of human abductive inner inferential processes, there are implicit routines, that is, unconscious cognitive performances that obviously cannot be contemplated by a formal model such as the GW-Schema. Peirce already indicated this aspect when illustrating the role of instinct [24] (8.223) and of what Galileo named the lume naturale [24] (6.477), a kind of an innate inclination for guessing right: we have to remember, following a Peircean basic insight concerning abduction, that this kind of reasoning is constitutively " akin to the truth": "It is a primary hypothesis underlying all abduction that the human mind is akin to the truth in the sense that in a finite number of guesses it will light upon the correct hypothesis" [24] (7.220). This and other cognitive and epistemological features can

13 Hintikka disapproves this Peircean employment of the word "induction": "I do not think that it is instructive to call such reasoning inductive, but this is a merely terminological matter" [40] (pp. 52 and 55). 
be described in more detail thanks to the eco-cognitive model (EC-Model) of abduction that will be summarized in the following final two subsections.

\subsection{The Eco-Cognitive Model of Abduction}

Condition 9 of the GW-schema (cf. previous subsection) is really puzzling. Indeed, it is not said that consistency and minimality constraints have to possess a privileged status, even if they have been emphasized in the "standard view" (AKM model, see the previous subsection) of abduction proposed by various classical logical accounts, more inclined to deal with selective abduction [38] —for example, in diagnostic cognitions (in which abduction is mainly seen as an inferential process of "selecting" from a "repository" of pre-stored hypotheses) than with creative abduction (abduction that produces new hypotheses). ${ }^{14}$

For example, the consistency requirement is questioned by the banal fact that, as Paul Feyerabend observes in Against Method [41], contradiction plays a fundamental role in generating creative hypotheses, that is what I have called creative abductive cognition. In these cases, there is a kind of "counterrule" which works against consistency and "[...] advises us to introduce and elaborate hypotheses which are inconsistent with well-established theories and/or well-established facts" [41] (p. 20). This rule also implies that creating "alternatives" is a good policy because "proliferation of theories is beneficial for science".

Moreover, clause 9 in the GW-model is related to two problems: (1) determining criteria for hypothesis selection and (2) building appropriate conditions for thinking up potential candidate hypotheses for the selection itself. Woods labels the first the "cutdown" problem and the second the "fill-up problem". It is this twofold problem that indicates the main equivocalness of the ignorance-preserving character of abduction contended by Gabbay and Woods: indeed, we have to state that the inferential procedures of generation (fill-up) and of selection (cutdown) can both be enough - even when a standard inductive evaluation moment lacks-to activate and accept [clause (11) of the GW-schema] an abductive hypothesis, and so to reach important knowledge results. In this case, the results are the fruit of a knowledge enhancing activity, as I have extendedly illustrated in $[32,42,43]$ : the instrumental components (which permit one's target to be reached) promote both abductive generation and abductive selection, and it is not needed that they should deal with classical plausibilistic worries, such as consistency and minimality.

In these knowledge enhancing cases, the best selection is accomplished in the absence of the experimental trial (which is instead basically characterized by the received view of abduction in terms of the well-known so-called "inference to the best explanation"). In a certain sense, the generation process alone can be sufficient: this is, for example, patent in the case of human perception, where the produced hypothesis is instantaneous and singular. We have to remember that perception was seen by Peirce as an "abductive" quick and ungoverned (and so automatic) process of production of knowledge. Perception, in the Peircean speculative light, is presented as a carrier for the immediate recovery of knowledge that was previously elaborated in our mind through more complicated inferential procedures. Peirce says: "Abductive inference shades into perceptual judgment without any sharp line of demarcation between them" [44] (p. 304). ${ }^{15}$

The idea of abductive cognition as perception immediately indicates central aspects of what I called its eco-cognitive character because, in this case, individual human agents, sensorial apparatuses, environment, and cognition are all regarded. To better grasp my eco-cognitive model of abduction, we must make a reference to the recent cognitive science studies on embodied and distributed cognitive systems: in my view of abductive cognition, the singular "practical agent" plays a dominant

14 I have suggested the distinction between selective and creative abduction in [38].

15 In [8] and [32], I have further illustrated the Peircean philosophical idea of perception as a case of abduction by showing how it can be corroborated thanks to recent cognitive research, also related to AI. 
role, an agent that is operating "on the ground", that is, in the situations of everyday actual life. The original work in distributed cognition was first of all related to the conviction that cognition is a socially distributed process, which is occurring in real practices. Cognitive processes are situated in and distributed across actual socio-artifactual contexts, against the standard view, inherited by the philosophical and psychological tradition, which privileged internalism and assigned to external representations, cognitive delegations, and collaborative processes a mere ancillary role. An ecological view helps seeing the central role in cognition of the agent-environment interplay: to make an example, in collective work environments, human beings and artifactual technologies are intertwined in manipulating representations, for example with the target of solving problems. It is exactly in this complex-socially, materially and temporally-distributed interplay that we can grasp the real structure of cognitive processes.

The theory of distributed cognition was proposed by Edwin Hutchins to analyze common problem-solving routines in actual human situations, but the theory soon acquired an important relevance for the entire research in cognitive science. In his well-known book Cognition in the Wild [45], Hutchins illustrates how agents exploit tools, props, and instruments (and, at the same time, external cognitive representations) to create, build, manipulate, and preserve representations. Hutchins thinks that the cognitive characters and properties of a distributed cognitive system is strictly connected with specific physical and "material" properties of the external representational mediators which are exploited. ${ }^{16}$

My eco-cognitive model of abduction (EC-model) ${ }_{1}^{17}$ has to be seen in the cognitive perspective I have just described. In the various situations in which we see abduction at work, from the ideally logical and mathematical to the more or less empirical, I insistently stress the eco-cognitive character of abduction. Reasoning is something realized by cognitive systems and, as an approximation, a cognitive system is a triple $(A, T, R)$, in which $A$ is an agent, $T$ is a cognitive target of the agent, and $R$ refers to the cognitive resources which the agent can exploit during the process aimed at reaching a target, for example, information, time and computational capacity, to recall the most relevant. Hence, my practical agents are embodied distributed cognitive systems: cognition is embodied and the interplay between brains, bodies, and external environment constitutes its dominant aspects. Cognition happens thanks to a continuous flow of information in a complicated distributed system that is occurring in the intertwining of humans, artifacts, and the environment, in which instinctual and subliminal capacities also play a relevant role. I have to stress that this interplay is especially important and dominant in several forms of abductive cognition.

I have said above, just making an example among the many, that perception can be seen as an abduction in which a fast and uncontrolled knowledge production is at work. Consequently, this means that-at least in this wider perspective-GW-schema is not canonical for abduction. The schema refers to what I have called "sentential abduction" [22] (Chapter 1), that is, abduction performed by symbols conveying propositional content. In this schema, it is impossible to insert cases of abductive cognition such as, for example, perception or the production of models (cf. [42]) in scientific discovery.

This theoretical framework, I have to remember, is also coherent with Peirce's philosophical major tenets: he thought that the concept of "inference" has to be intended semiotically, in a wide sense, (and not merely "logically"): he clearly says that all inference is a form of sign activity, where the word sign includes "feeling, image, conception, and other representation" [24] (5.283). Indeed, I think that this Peircean semiotic framework can be fruitfully intertwined with my perspective on cognitive systems as embodied and distributed systems. On the contrary, the GW-Schema refers, even if in a very effective way, to that subset of cognitive systems abductive processes that are realized thanks to explicit propositional contents. I think Woods agrees with me: "[...] the GW-model helps get us

16 For a deeper illustration of this interaction and of the role played by external representations as "material anchors for conceptual blends" [46] is a mandatory reference. A summary of the so-called "cognitive ecology" is illustrated in [47].

17 A first characterization of this model has been given in my book [22]. 
started in thinking about abduction, but it is nowhere close, at any level of abstraction, to running the whole show. It does a good job in modelling the ignorance-preserving character of abduction; however, since it leaves the $S_{i}$ of the schema's clause $(T)$ unspecified, it makes little contribution to the fill-up problem" [48] (p. 244).

Finally, it is important to stress that in my extended eco-cognitive framework the cutdown and fill-up problems in abductive cognition turn out to be stunningly related to the contexts. We can abduce a concept or a model when making science, where rationality is at play, but we can also abduce a hypothesis (a fictional character for example) in literature, or in moral cognition (the choice of a hypothetical judgment to motivate moral actions). We say that in these situations abductive hypotheses are basically evidentially inert and so admitted and actuated as a basis for further knowledge and/or action. In summary, the eco-cognitive research on abduction motivates a wide approach to "good reasoning", which certainly favors further studies on new "naturalized" logics of abductive cognition. In this way, we can overcome the average typical attitude of traditional logicians: it has been typical of them to manifest disinterest in abduction and other forms of third-type reasoning, as I have explained above, a skeptical attitude about unorthodox ways of good reasoning.

\subsection{Abduction Naturalized: The Importance of Eco-Cognitive Situatedness}

A good abductive logical system can be standardly illustrated by the following levels, which refer to the fill-up and the cutdown aspects:

- a base logic $L_{1}$ with demonstration procedures $\Pi$;

- $\quad$ an abductive algorithm which exhibits $\Pi$ to look for missing premisses and other formulas to be abduced;

- a logic $L_{2}$ for deciding which abduced formulas can be chosen, the criteria and methods of selection, etc. This logic is associated to the indication of suitable constraints concerning consistency, plausibility, relevance (topical, full-use, irredundancy-oriented, probabilistic), etc., and economy, making the ideal agent able to discount and select information which does not resolve the task at stake [23] (we have also observed above that other more instrumental criteria-and not merely consistency, plausibility, and similar ones-can be at work in strong cases of creative abduction).

The EC-model of abduction immediately suggests that, if we aim at naturalizing the logic of the abductive inferences and of its particular consequence relation, which indeed must be powerfully "eco-cognitive-sensitive", the following requirements are mandatory:

1. optimization of situatedness: situatedness is related to eco-cognitive aspects. To promote the solution of an abductive problem starting data and cognitions involved in the production of new hypotheses have to be seen as optimally positioned;

2. this optimality is rendered possible by a maximization of changeability of the flux of information available to the abducer (initial data) but also of the generated hypotheses that have to be various but-fundamentally—optimally "excogitated";

3. therefore, abductive inferential procedures are extremely information-sensitive, that is, the flow of information which affects them is uninterrupted and human (or machine)-boosted and enriched when necessary. This is not the case of demonstrations in classical logic, in which the adjustments of the inputs are minimized, demonstrations are considered with "given" and relatively stable inputs, and the burden of demonstrations is dominant and assigned to the inference rules, and to the strategic selection of them together with the selection of their suitable sequentiality (see [32] (Chapter 7, Section 7.2));

4. indeed, in an eco-cognitive perspective, an abductive "inferential problem" can be enhanced by the emergence of new information in a temporal dimension that favors the restarting of the inferential process itself. In the case of this cycle of reasoning, we are dealing with the so-called 
nonmonotonic character of abductive reasoning. Abductive consequence is characterized by new and newly appeared information, and so it is a defeasible kind of reasoning.

In summary, an appropriate naturalization of a logic of abduction has to consider the important role of the constant flux of information from the eco-cognitive surroundings in which the following two aspects count:

1. the new information available,

2. the new information inferentially generated.

Finally, let us stress again, as I have already indicated, the relevance of

- multimodality: the logical inferential procedure of adjustment of initial data must be clearly considered as multimodal, both from the perspective of cognitive tools "represented" (not only propositions, but also diagrams, or icons, for instance), and from the perspective of the applied rules that can be based on models (model-based). In addition, possible algorithmic computational components have to be considered pertinent.

In conclusion, optimization of situatedness is the major feature of logical abductive inference, which basically overcomes the other properties such as minimality, consistency, relevance, plausibility, etc. I have indicated above. These are special subsets of optimization processes, which refer to the particular type of situatedness required. I have often stressed in my studies the crucial case of abductions in science, in which the optimization of eco-cognitive situatedness has to deal with various constraints, which fundamentally regard the importance of the so-called epistemic virtues. ${ }^{18}$ Some examples of epistemic virtues are: hearing various sides of the scientific "stories" during the interplay among various scientists, but also among scientists and artifacts, and scientists and results coming from the experiments; open-mindedness; tolerance; impartiality; carefulness and sensitiveness to details; "deference" to the evidence (avoiding to change at will the starting data and the involved rules); being willing to question assumptions; giving and asking for reasons (applying suitable rules and verifying their rightness); being curious; being intellectually brave-that is, not merely believing what it is convenient to believe; etc.

In the final chapter of my last book on abduction [32], I have also stressed that constraints, methods, cognitive virtues, etc., which are concerned in scientific reasoning, on one side depict "limitations" of the cognitive behaviors involved, limitations that otherwise are fruitful because on the other side they also incorporate a commitment to the preservation of that optimization of eco-cognitive openness, which uniquely can permit the prospering of "human" abductive creativity in science.

\section{Conclusions}

In this article, I have analyzed and criticized some basic tenets of the John Woods' book on "errors of reasoning", and my own perspectives concerning abduction, to promote the project of the so-called naturalization of logic. I have suggested a criticism of the tradition of mainstream logic able to favor a new collaboration between logic and cognitive science, doing for logic what, many decades ago, philosophers such as Quine have proposed for epistemology: a "naturalization" of the logic of human reasoning. I have also illustrated that naturalizing logic requires an agent-based approach, a reconsideration of the cognitive status of fallacies (and a vindication of their positive inferential role), and the acceptance of a new eco-cognitive framework, also intertwined with the recent studies in the area of distributed cognition. To assist the reader in appreciating the naturalization of logic, I have also afforded the problem of the exceptional positive cognitive importance of the famous fallacy of the affirming the consequent (that is abduction). I have contended that abduction is not

18 I have described this problem of moral epistemology in [32] (Chapter 6, Section 6.1.2). 
only an ignorance-preserving type of cognition, as Woods contends, but also a knowledge enhancing one. To corroborate this result, I have synthetically described my own eco-cognitive model (EC-model) of abduction, which also explains that, thanks to abduction, knowledge can be enhanced, even when abduction is not taken as an inference to the best explanation in the standard sense, that is, an inference that also involves an inductive (so-called by Peirce) empirical process of evaluation. Finally, the last part of the article provides an analysis of the importance of the optimization of situatedness, a concept which is necessary to understand that "maximization of abducibility", which is distinctive of modern science cognitive endowments.

Funding: This research was funded by Blue Sky Research 2017-University of Pavia, Pavia, Italy.

Acknowledgments: For the informative critiques and interesting exchanges that assisted me to enrich my analysis of the naturalization of logic and/or abductive cognition, I am obligated to John Woods, Atocha Aliseda, Woosuk Park, Luís Moniz Pereira, Paul Thagard, to the three reviewers, and to my collaborators Tommaso Bertolotti and Selene Arfini.

Conflicts of Interest: The author declares no conflict of interest. The funders had no role in the design of the study; in the collection, analyses, or interpretation of data; in the writing of the manuscript, or in the decision to publish the results.

\section{References}

1. Woods, J. Errors of Reasoning. Naturalizing the Logic of Inference; College Publications: London, UK, 2013.

2. Dewey, J. The Later Works; Southern Illinois Press: Carbondale, IL, USA, 1981-1991.

3. Toulmin, S. The Uses of Argument; Cambridge University Press: Cambridge, UK, 1958.

4. Quine, W.V. Epistemology Naturalized. In Ontological Relativity and Other Essays; Columbia University Press: New York, NY, USA, 1969; pp. 69-90.

5. Finocchiaro, M. Arguments about Arguments; Cambridge University Press: Cambridge, UK, 2005.

6. Kowalski, R. Computational Logic and Human Thinking: How to be Artificially Intelligent; Cambridge University Press: Cambridge, UK, 2011.

7. Stenning, K.; van Lambangen, M. Human Reasoning and Cognitive Science; MIT Press: Cambridge, MA, USA, 2012.

8. Magnani, L. Naturalizing Logic. Errors of reasoning vindicated: Logic reapproaches cognitive science. J. Appl. Log. 2015, 13, 13-36. [CrossRef]

9. Byrne, R.M.J. The Rational Imagination: How People Create Alternatives to Reality; MIT Press: Boston, MA, USA, 2007.

10. Migliore, S.; Curcio, G.; Mancini, F.; Cappa, S.F. Counterfactual thinking in moral judgment: An experimental study. Front. Psychol. 2014, 5, 451. [CrossRef] [PubMed]

11. Saptawijaya, A.; Pereira, L.M. Towards modeling morality computationally with Logic Programming. In Proceedings of the 16th International Symposium on Practical Aspects of Declarative Languages (PADL'14), San Diego, CA, USA, 20-21 January 2014; Flatt, M., Guo, H., Eds.; Springer: Heidelberg/Berlin, Germany, 2014; pp. 104-119.

12. Pereira, L.M.; Saptawijaya, A. Modelling morality with prospective logic. In Machine Ethics; Anderson, M., Anderson, S.L., Eds.; Cambridge University Press: Cambridge, UK, 2011; pp. 398-421.

13. Han, T.A.; Saptawijaya, A.; Pereira, L.M. Moral reasoning under uncertainty. In Logic for Programming, Artificial Intelligence, and Reasoning-18; Springer: Berlin, Germany, 2012; Volume 7180, pp. 212-227.

14. Pereira, L.M.; Pinto, A.M. Reductio ad absurdum argumentation in normal logic programs. In Proceedings of the Workshop on Argumentation and Non-Monotonic Reasoning (ArgNMR'07), Tempe, AZ, USA, 19 February 2007; pp. 96-113.

15. Pereira, L.M.; Pinto, A.M. Adaptive reasoning for cooperative agents. In Proceedings of the 18th International Conference on Applications of Declarative Programming and Knowledge Management (INAP'09), Évora, Portugal, 5 November 2009; Abreu, S., Seipel, D., Eds.; Springer: Heidelberg/Berlin, Germany, 2011; pp. 102-116.

16. Pereira, L.M.; Dietz, E.; Hölldobler, S. An Abductive Reasoning Approach to the Belief Bias Effect. In Proceedings of the Fourteenth International Conference on Principles of Knowledge Representation and Reasoning, Vienna, Austria, 20-24 July 2014. 
17. Pereira, L.M.; Dietz, E.; Hölldobler, S. Contextual abductive reasoning with side-effects. Theory Pract. Log. Program. 2014, 14, 633-648. [CrossRef]

18. Alferes, J.J.; Knorr, M.; Wang, K. Forgetting under the well-founded semantics. In Logic Programming and Nonmonotonic Reasoning (Lecture Notes in Computer Science 8148); Cabalar, P., Son, T., Eds.; Springer: Berlin/Heidelberg, Germany, 2013; pp. 36-41.

19. Han, T.A. Intention Recognition, Commitment and Their Roles in the Evolution of Cooperation; Springer: Cham, Switzerland, 2013.

20. Han, T.A.; Pereira, L.M.; Santos, F.C.; Lenaerts, T. Emergence of Cooperation via Intention Recognition, Commitment, and Apology. A Research Summary. AI Commun. 2015, 28, 709-715. [CrossRef]

21. Peirce, C.S. Reasoning and the Logic of Things: the 1898 Cambridge Conferences Lectures by Charles Sanders Peirce; Ketner, K.L., Ed.; Harvard University Press: Amsterdam, The Netherlands, 2005.

22. Magnani, L. Abductive Cognition. The Epistemological and Eco-Cognitive Dimensions of Hypothetical Reasoning; Springer: Heidelberg/Berlin, Germany, 2009.

23. Gabbay, D.M.; Woods, J. The Reach of Abduction. In A Practical Logic of Cognitive Systems; North-Holland: Amsterdam, The Netherlands, 2005; Volume 2.

24. Peirce, C.S. Collected Papers of Charles Sanders Peirce; Harvard University Press: Cambridge, MA, USA, 1931-1958; Volumes 1-6, Hartshorne, C., Weiss, P., Eds.; Volumes 7-8, Burks, A.W., Ed.

25. Magnani, L. Are Heuristics Knowledge-Enhancing? Abduction, Models, and Fictions in Science. In Heuristic Reasoning; Ippoliti, E., Ed.; Springer: Heidelberg/Berlin, Germany, 2014; pp. 29-56.

26. Woods, J. The Death of Argument; Kluwer Academic Publishers: Dordrecht, The Netherlands, 2004.

27. Gigerenzer, G.; Todd, P. Simple Heuristics that Make Us Smart; Oxford University Press: Oxford, UK; New York, NY, USA, 1999.

28. Gigerenzer, G.; Selten, R. Bounded Rationality. The Adaptive Toolbox; The MIT Press: Cambridge, MA, USA, 2002.

29. Raab, M.; Gigerenzer, G. Intelligence as smart heuristics. In Cognition and Intelligence. Identifying the Mechanisms of the Mind; Sternberg, R.J., Prets, J.E., Eds.; Cambridge University Press: Cambridge, MA, USA, 2005; pp. 188-207.

30. Gigerenzer, G.; Brighton, H. Homo heuristicus: Why biased minds make better inferences. Top. Cogn. Sci. 2009, 1, 107-143. [CrossRef] [PubMed]

31. Brandom, R. Articulating Reason: An Introduction to Inferentialism; Harvard University Press: Cambridge, MA, USA; Edinburgh, UK, 2000.

32. Magnani, L. The Abductive Structure of Scientific Creativity. An Essay on the Ecology of Cognition; Springer: Cham, Switzerland, 2017.

33. Aliseda, A. Seeking Explanations: Abduction in Logic, Philosophy of Science and Artificial Intelligence. Ph.D. Thesis, Institute for Logic, Language and Computation, Amsterdam, The Netherlands, 1997.

34. Aliseda, A. Abductive Reasoning. Logical Investigations into Discovery and Explanation; Springer: Berlin, Germany, 2006.

35. Kowalski, R.A. Logic for Problem Solving; Elsevier: New York, NY, USA, 1979.

36. Kuipers, T.A.F. Abduction aiming at empirical progress of even truth approximation leading to a challenge for computational modelling. Found. Sci. 1999, 4, 307-323. [CrossRef]

37. Kakas, A.; Kowalski, R.A.; Toni, F. Abductive logic programming. J. Log. Comput. 1993, 2, 719-770. [CrossRef]

38. Magnani, L. Abduction, Reason, and Science. Processes of Discovery and Explanation; Kluwer Academic/Plenum Publishers: New York, NY, USA, 2001.

39. Meheus, J.; Verhoeven, L.; Van Dyck, M.; Provijn, D. Ampliative adaptive logics and the foundation of logic-based approaches to abduction. In Logical and Computational Aspects of Model-Based Reasoning; Magnani, L., Nersessian, N.J., Pizzi, C., Eds.; Kluwer Academic Publishers: Dordrecht, The Netherlands, 2002; pp. 39-71.

40. Hintikka, J. Socratic Epistemology. Explorations of Knowledge-Seeking by Questioning; Cambridge University Press: Cambridge, UK, 2007.

41. Feyerabend, P. Against Method; Verso: London, UK; New York, NY, USA, 1975.

42. Magnani, L. Scientific models are not fictions. Model-based science as epistemic warfare. In Philosophy and Cognitive Science. Western and Eastern Studies; Magnani, L., Li, P., Eds.; Springer: Heidelberg/Berlin, Germany, 2012; pp. 1-38. 
43. Magnani, L. Is Abduction Ignorance-Preserving? Conventions, Models, and Fictions in Science. Log. J. IGPL 2013, 21, 882-914. [CrossRef]

44. Peirce, C.S. Visual cognition and cognitive modeling. In Philosophical Writings of Peirce; Buchler, J., Ed.; Dover: New York, NY, USA, 1955; pp. 302-305.

45. Hutchins, E. Cognition in the Wild; The MIT Press: Cambridge, MA, USA, 1995.

46. Hutchins, E. Material anchors for conceptual blends. J. Pragmat. 2005, 37, 1555-1577. [CrossRef]

47. Hutchins, E. Cognitive ecology. Top. Cogn. Sci. 2010, 2, 705-715. [CrossRef] [PubMed]

48. Woods, J. Recent Developments in Abductive Logic. Stud. Hist. Philos. Sci. 2011, 42, 240-244. [CrossRef]

(C) 2018 by the authors. Licensee MDPI, Basel, Switzerland. This article is an open access article distributed under the terms and conditions of the Creative Commons Attribution (CC BY) license (http://creativecommons.org/licenses/by/4.0/). 